\title{
Tridimensional Observations of Nearby Active Galaxies Using OAO Spectronebulagraph
}

\author{
M. Yoshida(1), Y. Shimizu(1) ${ }^{(1)}$ H. Koyano(1), G. Kosugi ${ }^{(2)}$, K. Aoki ${ }^{(2)}$, \\ H. Ohtani ${ }^{(2)}$, T. Sasaki ${ }^{(3)}$, M. Sasaki $^{(4)}$ \\ (1) Okayama Astrophysical Observatory, Kamogata-cho, Asakuchi-gun, \\ Okayama 719-02, Japan \\ (2) Department of Astronomy, Kyoto University, Kyoto 606-01, Japan \\ (3) National Astronomical Observatory, Mitaka, Tokyo 181, Japan \\ (1) Shimonoseki City University, Daigaku-cho, Shimonoseki, \\ Yamaguchi 751, Japan
}

\section{Introduction}

We developed a slit-scanning type 3D-spectroscopic system named as Spectronebulagraph (hereafter SNG; Kosugi et al. 1994) at Okayama Astrophysical Observatory. A detailed description about SNG is presented by Ohtani et al. elsewhere in this colloquium. We present here the results of the 3D-spectroscopy of four nearby active galaxies by using SNG.

\section{NGC 4449}

This nearby (at distance of 5.4 Mpc) Magellanic irregular galaxy has many $\mathrm{H}$ II regions in its main body suggesting violent star-formation activity. We performed tridimensional observations of NGC 4449 using SNG in order to obtain detailed kinematic structure and then to investigate the star-formation mechanism of NGC 4449. H $\alpha$ intensity map, $\mathrm{H} \alpha$ velocity field are shown in figure 1. As is obvious in lower panel of figure 1, no global rotational motion was detected. Most impressive feature of the velocity field is kpc-scale mosaic structure of low velocity and high velocity components. Also, many filamentary components were newly found in 3D-datacube of the SNG observation.

\section{NGC 3646}

This large, massive, and very gas-rich galaxy is characterized by bright, extraordinary large ring with the diameter of approximately $40 \mathrm{kpc}$. In spite of its peculiarity, no detailed observational study had been made on this object. SNG observations of NGC 3646 revealed that its ring is very bright in $\mathrm{H} \alpha$ suggesting that violent star-formation occurs. The velocity field obtained by SNG suggested that the ring is expanding outward with the velocity of $30 \mathrm{~km} \mathrm{~s}^{-1}$ and that the gas behind the ring is contracting to the center. These observational results of NGC 3646 are almost consistent with the results of the numerical models of the formation of the ring galaxy made by Struck-Marcell and Appleton (1987). 


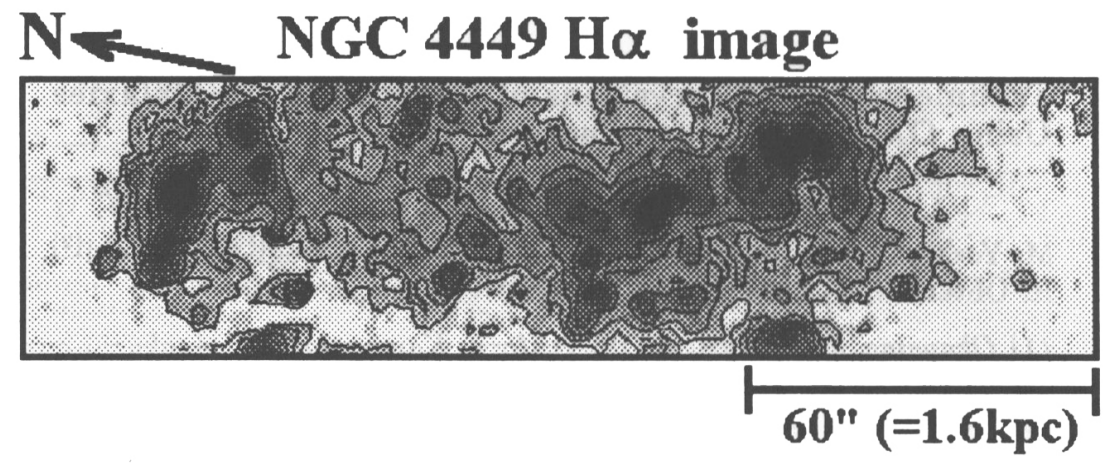

NGC $4449 \mathrm{H} \alpha$ velocity field

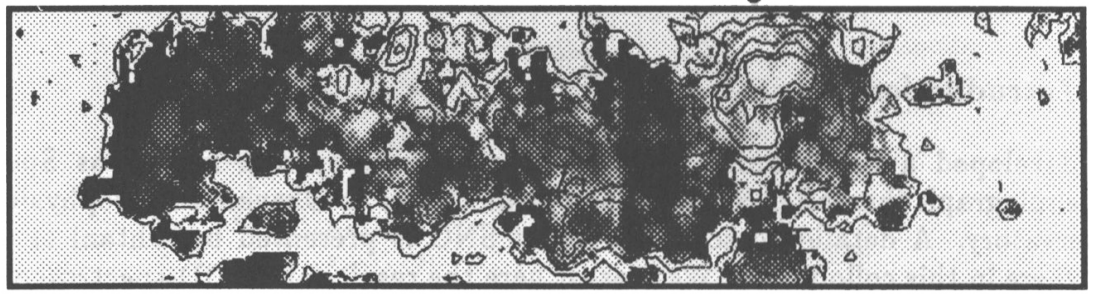

$210 \mathrm{~km} / \mathrm{s}$

$265 \mathrm{~km} / \mathrm{s}$

Figure 1. (upper panel) Ha line intensity map of NGC 4449. (lower panel) $\mathrm{H} \alpha$ velocity field (gray scale) of NGC 4449 superimposed on its $\mathrm{H} \alpha$ line intensity map (contour plot).

\section{M 82 and NGC 1275}

We performed near-infrared [S III] $\lambda \lambda 9069,9531$ observations of the well-studied proto-typical starburst galaxy $M 82$ in order to investigate the kinematics and excitation of the ionized gas in the inner region of the galaxy. Although the rotational component is dominant in the kinematics of the central region of $M$ 82 , radial flow component is remarkable along the minor axis of the galaxy. The line width of [S III] becomes wider with distance from the nucleus along the minor axis of the galaxy.

The $\mathrm{H} \alpha,[\mathrm{N}$ II], [S II] and [O I] images of the Seyfert galaxy NGC 1275 was obtained by SNG. [O I] is intensely emitted everywhere in the ionized region of NGC 1275. The distribution of the excitation of the ionized gas is patchy. There are some compact regions that show high $[\mathrm{O} \mathrm{I}] / \mathrm{H} \alpha$ line intensity ratio near the nucleus. On the other hand, the region in which $[0 \mathrm{I}]$ is significantly weak relative to $\mathrm{H} \alpha$ exists at $10^{\prime \prime}$ west of the nucleus. The high $[\mathrm{O} \mathrm{I}] / \mathrm{H} \alpha$ regions other than the Seyfert nucleus may be the regions in which strong shock occurs or those irradiated by UV-X ray from the cooling flow. The low $[\mathrm{O} \mathrm{I}] / \mathrm{H} \alpha$ region located at $10^{\prime \prime}$ west of the nucleus possibly be a bright $\mathrm{H}$ II region in the cooling flow. 